\title{
Dipolar interaction induced band gaps and flat modes in surface-modulated magnonic crystals
}

\author{
R. A. Gallardo, ${ }^{1,6}$ T. Schneider, ${ }^{2,3}$ A. Roldán-Molina, ${ }^{4}$ M. Langer, ${ }^{2,5,7}$ J. Fassbender, ${ }^{2}$ K. Lenz, ${ }^{2}$ J. Lindner, ${ }^{2}$ and P. Landeros ${ }^{1,6}$ \\ ${ }^{1}$ Departamento de Física, Universidad Técnica Federico Santa María, Avenida España 1680, Valparaíso, Chile \\ ${ }^{2}$ Institute of Ion Beam Physics and Materials Research, Helmholtz-Zentrum Dresden-Rossendorf, \\ Bautzner Landstrasse 400, 01328 Dresden, Germany \\ ${ }^{3}$ Department of Physics, Technische Universität Chemnitz, Reichenhainer Strasse 70, 09126 Chemnitz, Germany \\ ${ }^{4}$ Universidad de Aysén, Calle Obispo Vielmo 62, Coyhaique, Chile \\ ${ }^{5}$ Institute for Physics of Solids, Technische Universität Dresden, Zellescher Weg 16, 01069 Dresden, Germany \\ ${ }^{6}$ Center for the Development of Nanoscience and Nanotechnology (CEDENNA), 917-0124 Santiago, Chile \\ ${ }^{7}$ Swiss Light Source, Paul Scherrer Institute, 5232 Villigen, Switzerland
}

(Received 29 September 2017; published 10 April 2018)

\begin{abstract}
Theoretical results for the magnetization dynamics of a magnonic crystal formed by grooves on the surface of a ferromagnetic film, called a surface-modulated magnonic crystal, are presented. For such a system, the role of the periodic dipolar field induced by the geometrical modulation is addressed by using the plane-wave method. The results reveal that, under the increasing of the depth of the grooves, zones with magnetizing and demagnetizing fields act on the system in such a way that magnonic band gaps are observed in both Damon-Eshbach and backward volume geometries. Particularly, in the backward volume configuration, high-frequency band gaps and lowfrequency flat modes are obtained. By taking into account the properties of the internal field induced by the grooves, the flattening of the modes and their shift towards low frequencies are discussed and explained. To test the validity of the model, the theoretical results of this work are confirmed by micromagnetic simulations, and good agreement between both methods is achieved. The theoretical model allows for a detailed understanding of the physics underlying these kinds of systems, thereby providing an outlook for potential applications on magnonic devices.
\end{abstract}

DOI: 10.1103/PhysRevB.97.144405

\section{INTRODUCTION}

Spin waves (SWs) at microwave frequencies are of current interest for the field of nanomagnetism and spintronics. From a practical point of view, experimental techniques involving SWs allow us to determine the fundamental parameters of magnetic materials, while from the application viewpoint, such waves operate at gigahertz frequencies with nanometric wavelengths, which encourages the development of miniaturized microwave signal processing SW-based devices [1,2]. One of the potential aspects of SW-based technologies is that both the amplitude and the phase of spin waves may encode information $[3,4]$. In addition, SWs have further been proposed as building blocks for computational architectures allowing us to perform logic operations [5-7]. In the field of magnonics, artificial magnetic media with periodic properties, called magnonic crystals (MCs) $[1,2,8]$, are of great interest for magnon-based applications since their dynamic behavior significantly differs from those of non-periodic magnetic systems [9-17]. SWs in MCs exhibit adjustable frequency band gaps (BGs), which can be optimized by modulating the magnetic parameters or changing the geometry and arrangement of periodic scattering centers [9-33]. The design of MCs has wide versatility since these media can be created by artificial modulation of the magnetic properties, either in the form of dipolarly coupled nanowires [21], bicomponent magnonic crystals [12-14], width-modulated waveguides [30,32], antidot lattices [34,35], or modulated thickness films [36-41] or by means of ion implantation [42,43]. Also, reconfigurable magnonic crystals with a periodic induction of perpendicular magnetic anisotropy have been designed [44].
The broadband vector network analyzer ferromagnetic resonance (VNA-FMR) technique has been used to study the dynamic properties of magnonic crystals $[38,41,45,46]$, where additional modes have been observed in the backward-volume (BV) configuration due to the coupling of the coherent FMR mode with standing spin waves that have a wavelength that is an integer multiple of the periodicity. On the other hand, a large number of studies based on Brillouin light scattering have been carried out on MCs [11-14], where the presence of frequency band gaps has been confirmed, in full agreement with theoretical results. Moreover, bicomponent MCs have been widely studied [12-14,47-49], where periodic properties originate from a different saturation magnetization $M_{\mathrm{s}}$, anisotropy $K$, or exchange constant $A$. Thus, modification of these parameters allows for controlling the BG position and the localization of SWs. For instance, increasing the contrast of $M_{\mathrm{S}}$ of a bicomponent MC can lead to a broadening of the $\mathrm{BG}$ frequency range and enable the concentration of a spin-wave excitation within the zone of lower or higher saturation magnetization. However, experimentally defining material parameters such as magnetization or exchange length with laterally well defined periodicities often is not straightforward and suffers from limitations of the range in which variations are possible for a given material. A periodic surface modulation of a ferromagnetic (FM) thin film is an interesting alternative to creating magnonic structures and devices, where the periodic properties are attributed to the size of the modulation [3841,45,46,50-55]. For instance, yttrium iron garnet (YIG) films with periodic arrays of micrometer-sized etched grooves have been synthesized [45,46,50,51,53-55], while FM films with nanometric grooves have been created by means of ion 
implantation [42,43] and electron-beam lithography [40]. In these kinds of systems, it is shown that the periodic pattern influences the SW dynamics in both Damon-Eshbach (DE) and BV geometries. Particularly, for BV spin waves, rejection bands have been observed $[45,51,55]$ which are almost independent of the wave vector [55]. These nearly flat modes were attributed to the fact that spins became dynamically pinned as a result of the demagnetizing field, which is expected to increase in the $\mathrm{BV}$ configuration [55].

In this paper, a surface-modulated magnonic crystal (SMMC), which is formed by an array of grooves placed on one of the surfaces of a FM film, is theoretically addressed by using the plane-wave method and micromagnetic simulations. The dipolar fields created by the trenches are analytically described, and both the static and dynamic dipolar magnetic fields are taken into account. It is shown that the periodic dipolar fields open frequency band gaps in both DE and BV configurations. Particularly, in the BV geometry flat bands are obtained, which reveal a notorious frequency separation between them. The nature of the flatness of the modes and their shift towards low frequencies are explained by taking into account the spatial distribution of the internal field and the spin-wave localization. By controlling the depth of the grooves, the mode frequencies, spatial profiles, and forbidden frequency gaps of spin waves can be manipulated.

\section{THEORETICAL DESCRIPTION}

In a bicomponent $\mathrm{MC}$, the periodic properties originate from the contrast between two ferromagnetic materials with different magnetic parameters, for instance, the saturation magnetization $M_{\mathrm{s}}$ or exchange constant $A$. Nevertheless, in an surface-modulated MC the periodic properties arise mainly from the magnetic charges created at the edges of the stripelike grooves, as shown Fig. 1(b) for a one-dimensional SMMC. For a periodic magnetic system with infinite sides, some theoretical approaches have been used, such as the planewave method [22], the Green's function method [56], the multiple-scattering theory [57], the two-magnon scattering approach [36,38], and the Kronig-Penney model [18], among others. For finite periodic structures, where the boundary conditions at the system's edges are relevant, some theoretical approaches have also been developed [54,58-60]. For the system shown in Fig. 1, the plane-wave method will be applied.

The temporal evolution of the system can be described using the Landau-Lifshitz (LL) equation $\dot{\mathbf{M}}(\mathbf{r} ; t)=-\gamma \mathbf{M}(\mathbf{r} ; t) \times$ $\mathbf{H}^{\mathrm{e}}(\mathbf{r} ; t)$. Here, $\gamma$ is the absolute value of the gyromagnetic ratio, $\mathbf{M}(\mathbf{r} ; t)$ is the magnetization, and $\mathbf{H}^{\mathrm{e}}(\mathbf{r} ; t)$ is the effective field. For small deviations around the equilibrium, both the magnetization and the effective field are written as $\mathbf{M}(\mathbf{r} ; t)=$ $M_{\mathrm{s}} \hat{Z}+\mathbf{m}(\mathbf{r} ; t)$ and $\mathbf{H}^{\mathrm{e}}(\mathbf{r} ; t)=\mathbf{H}^{\mathrm{e} 0}(\mathbf{r})+\mathbf{h}^{\mathrm{e}}(\mathbf{r} ; t)$, respectively. Note that $\hat{Z}$ points along the equilibrium orientation of the magnetization, which lies in plane, and $\mathbf{h}^{\mathrm{e}}(\mathbf{r} ; t)$ is proportional to the dynamic magnetization $\mathbf{m}(\mathbf{r} ; t)$. Thus, in the linear regime, the LL equation reads

$$
\begin{gathered}
i \Omega m_{X}(\mathbf{r})=-m_{Y}(\mathbf{r}) H_{Z}^{\mathrm{e} 0}(\mathbf{r})+M_{\mathrm{s}} h_{Y}^{\mathrm{e}}(\mathbf{r}), \\
i \Omega m_{Y}(\mathbf{r})=m_{X}(\mathbf{r}) H_{Z}^{\mathrm{e} 0}(\mathbf{r})-M_{\mathrm{s}} h_{X}^{\mathrm{e}}(\mathbf{r}),
\end{gathered}
$$

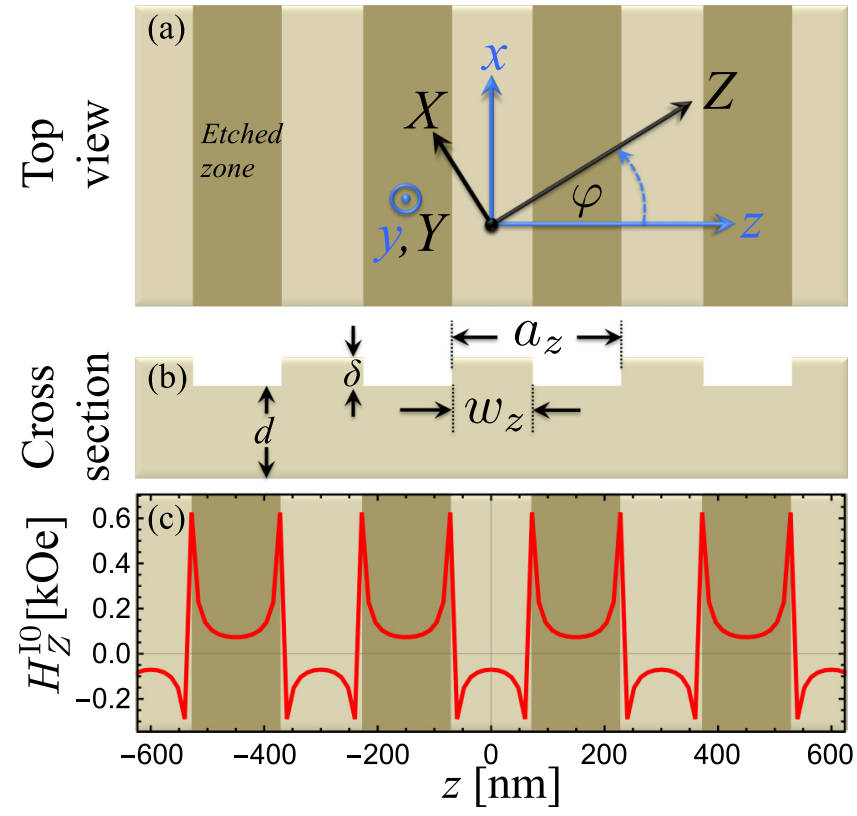

FIG. 1. In (a) the top view of the surface-modulated MC is shown, where two coordinate systems are depicted. The coordinates $(x, y, z)$ are defined by the periodic structure's geometry, while $(X, Y, Z)$ are defined according to the equilibrium orientation of the magnetization, which points along $Z$, with $X$ lying in the film's plane. In (b) the cross section is shown, where $d$ denotes the thickness of the nominal film, $\delta$ is the depth of the grooves, $a_{z}$ is the lattice parameter along the $z$ direction, and $w_{z}$ is the width of the thicker part of the film. The red curve in (c) shows the periodic dipolar field [see Eqs. (7) and (8)] created by the static magnetic charges at the edges of the grooves for $\delta=2 \mathrm{~nm}$ and $\varphi=0$. Additional magnetic parameters are given in Sec. III.

where it has been assumed $\mathbf{h}^{\mathrm{e}}(\mathbf{r} ; t)=\mathbf{h}^{\mathrm{e}}(\mathbf{r}) e^{i \omega t}$ and $\mathbf{m}(\mathbf{r} ; t)=$ $\mathbf{m}(\mathbf{r}) e^{i \omega t}$, and it is also defined $\Omega=\omega / \gamma$. Moreover, note that $H_{\eta}^{\mathrm{e} 0}\left(h_{\eta}^{\mathrm{e}}\right)$ is the $\eta$ component of the static (dynamic) effective magnetic field. The effective field is defined as $\mathbf{H}^{\mathrm{e}}(\mathbf{r})=\mathbf{H}+\mathbf{H}^{\mathrm{ex}}(\mathbf{r})+\mathbf{H}^{\mathrm{d}}(\mathbf{r})+\mathbf{H}^{\mathrm{I}}(\mathbf{r})$, where $\mathbf{H}$ is the external field and $\mathbf{H}^{\mathrm{ex}}(\mathbf{r})=4 \pi \lambda_{\mathrm{ex}}^{2} \nabla^{2} \mathbf{M}(\mathbf{r})$ is the exchange field with $\lambda_{\text {ex }}=\sqrt{2 A / 4 \pi M_{\mathrm{s}}^{2}}$, where $A$ is the exchange stiffness constant. Furthermore, $\mathbf{H}^{\mathrm{d}}(\mathbf{r})$ is the dipolar field of the flat film, and $\mathbf{H}^{\mathrm{I}}(\mathbf{r})$ is the dipolar field induced by the periodic magnetic charges that reside at the edges of the grooves $d<y<d+\delta$, where $d$ is the thickness of the nominal film and $\delta$ defines the depth of the grooves (see Fig. 1). According to Fig. 1, the periodic distribution of the top surface induces a periodic stray field that interacts with the magnetization of the nominal film. In this way, according to Bloch's theorem, the dynamic components of the magnetization can be expanded into Fourier series as $\mathbf{m}(\mathbf{r})=\sum_{\mathbf{G}} \mathbf{m}(\mathbf{G}) e^{i(\mathbf{G}+\mathbf{k}) \cdot \mathbf{r}}$, where $\mathbf{G}=G_{q} \hat{x}+G_{n} \hat{z}$ denotes a reciprocal lattice vector, with $G_{q}=\left(2 \pi / a_{x}\right) q, G_{n}=$ $\left(2 \pi / a_{z}\right) n$, and both $n$ and $q$ being integer numbers. Note that $a_{x}\left(a_{z}\right)$ represents the lattice constant, or lattice parameter, along the $x$ axis ( $z$ axis). The above picture considers a general two-dimensional periodic modulation of the grooves, which can easily be adapted to one-dimensional periodic structures by setting $G_{q}=0$. The dynamic components of the dipolar 
field averaged over the film's thickness are

$$
h_{Y}^{\mathrm{d}}(\mathbf{r})=-4 \pi \sum_{\mathbf{G}} m_{Y}(\mathbf{G}) \zeta(\mathbf{G}+\mathbf{k}, d) e^{i(\mathbf{G}+\mathbf{k}) \cdot \mathbf{r}}
$$

and

$$
h_{X}^{\mathrm{d}}(\mathbf{r})=4 \pi \sum_{\mathbf{G}} m_{X}(\mathbf{G}) \xi(\mathbf{G}, \mathbf{k})^{2} \frac{\zeta(\mathbf{G}+\mathbf{k}, d)-1}{|\mathbf{G}+\mathbf{k}|^{2}} e^{i(\mathbf{G}+\mathbf{k}) \cdot \mathbf{r}},
$$

where

$$
\zeta(\mathbf{Q}, d)=\frac{2 \sinh [|\mathbf{Q}| d / 2] e^{-|\mathbf{Q}| d / 2}}{|\mathbf{Q}| d}
$$

and

$$
\xi(\mathbf{G}, \mathbf{k})=\left(G_{n}+k_{z}\right) \sin \varphi-\left(G_{q}+k_{x}\right) \cos \varphi .
$$

Likewise, the dynamic exchange field components are

$$
h_{X, Y}^{\mathrm{ex}}(\mathbf{r})=-4 \pi \lambda_{\mathrm{ex}}^{2} \sum_{\mathbf{G}}(\mathbf{G}+\mathbf{k})^{2} m_{X, Y}(\mathbf{G}) e^{i(\mathbf{G}+\mathbf{k}) \cdot \mathbf{r}} .
$$

The $Z$ component of the static dipolar field created by the edges of the etched zones can be written as $H_{Z}^{\mathrm{I} 0}(\mathbf{r})=H_{1 Z}^{\mathrm{I} 0}(\mathbf{r})+$ $H_{2 Z}^{\mathrm{I} 0}(\mathbf{r})$, with $H_{1 Z}^{\mathrm{I} 0}(\mathbf{r})\left[H_{2 Z}^{\mathrm{I} 0}(\mathbf{r})\right]$ being the static field inside the zone $d>y>0[d+\delta>y>d]$. These components are (see details in Appendix A)

$$
H_{1 Z}^{\mathrm{I} 0}(\mathbf{r})=-2 \pi M_{\mathrm{s}} \sum_{\mathbf{G}} C_{\mathbf{G}} \chi(\mathbf{G})^{2} \frac{\eta(\mathbf{G}) d}{|\mathbf{G}|^{2}(d+\delta)} e^{i \mathbf{G} \cdot \mathbf{r}}
$$

and

$$
H_{2 Z}^{\mathrm{I} 0}(\mathbf{r})=4 \pi M_{\mathrm{s}} \sum_{\mathbf{G}} C_{\mathbf{G}} \chi(\mathbf{G})^{2} \delta \frac{\zeta(\mathbf{G}, \delta)-1}{|\mathbf{G}|^{2}(d+\delta)} e^{i \mathbf{G} \cdot \mathbf{r}},
$$

where

$$
\eta(\mathbf{G})=\frac{e^{-|\mathbf{G}|(d+\delta)}}{|\mathbf{G}| d}\left(e^{|\mathbf{G}| d}-1\right)\left(e^{|\mathbf{G}| \delta}-1\right) .
$$

Also, the dynamic components of the field $\mathbf{H}^{\mathrm{I}}(\mathbf{r})$ are

$$
\begin{aligned}
h_{Y}^{\mathrm{I}}(\mathbf{r})= & 2 \pi \sum_{\mathbf{G}, \mathbf{G}^{\prime}} C_{\mathbf{G}^{\prime}} e^{i\left(\mathbf{G}+\mathbf{G}^{\prime}+\mathbf{k}\right) \cdot \mathbf{r}}\left\{m_{Y}(\mathbf{G}) \eta\left(\mathbf{G}+\mathbf{G}^{\prime}+\mathbf{k}\right)\right. \\
& \left.-i m_{X}(\mathbf{G}) \xi\left(\mathbf{G}+\mathbf{G}^{\prime}, \mathbf{k}\right) \frac{\eta\left(\mathbf{G}+\mathbf{G}^{\prime}+\mathbf{k}\right)}{\left|\mathbf{G}+\mathbf{G}^{\prime}+\mathbf{k}\right|}\right\}
\end{aligned}
$$

and

$$
\begin{aligned}
h_{X}^{\mathrm{I}}(\mathbf{r})= & -2 \pi \sum_{\mathbf{G}, \mathbf{G}^{\prime}} C_{\mathbf{G}^{\prime}} e^{i\left(\mathbf{G}+\mathbf{G}^{\prime}+\mathbf{k}\right) \cdot \mathbf{r}} \\
& \times\left\{m_{X}(\mathbf{G}) \xi\left(\mathbf{G}+\mathbf{G}^{\prime}, \mathbf{k}\right)^{2} \frac{\eta\left(\mathbf{G}+\mathbf{G}^{\prime}+\mathbf{k}\right)}{\left|\mathbf{G}+\mathbf{G}^{\prime}+\mathbf{k}\right|^{2}}\right. \\
& \left.+i m_{Y}(\mathbf{G}) \xi\left(\mathbf{G}+\mathbf{G}^{\prime}, \mathbf{k}\right) \frac{\eta\left(\mathbf{G}+\mathbf{G}^{\prime}+\mathbf{k}\right)}{\left|\mathbf{G}+\mathbf{G}^{\prime}+\mathbf{k}\right|}\right\} .
\end{aligned}
$$

In Eqs. (7), (8), (10), and (11) the coefficients $C_{\mathbf{G}}$ account for the geometry of the periodic structure, which may be in the form of stripes, circular dots, squares, etc. [38]. In general, the static field component $H_{Z}^{\mathrm{I} 0}(\mathbf{r})$ and the dynamic one $h_{X, Y}^{\mathrm{I}}$ enter directly in the dynamics of the system through Eq. (1), while the $H_{X}^{\mathrm{I0}}(\mathbf{r})$ and $H_{Y}^{\mathrm{I0}}(\mathbf{r})$ components affect only the static properties of the system. Now, inserting all field contributions into Eq. (1), the following eigenvalue problem is obtained:

$$
\tilde{\mathbf{A}} \mathbf{m}_{\mathbf{G}}=i \Omega \mathbf{m}_{\mathbf{G}},
$$

where $\mathbf{m}_{\mathbf{G}}^{\mathrm{T}}=\left[m_{X}\left(G_{1}\right), \ldots, m_{X}\left(G_{N}\right), m_{Y}\left(G_{1}\right), \ldots, m_{Y}\left(G_{N}\right)\right]$ is the eigenvector and $\tilde{\mathbf{A}}$ is given by

$$
\tilde{\mathbf{A}}=\left(\begin{array}{ll}
\tilde{\mathbf{A}}^{X X} & \tilde{\mathbf{A}}^{X Y} \\
\tilde{\mathbf{A}}^{Y X} & \tilde{\mathbf{A}}^{Y Y}
\end{array}\right) .
$$

Explicit expressions of the matrix elements of Eq. (13) can be found in Appendix B. Now, by using standard numerical methods and a convergence test to check the reliability of the results, the eigenvalues and eigenvectors of Eq. (12) can be obtained.

Additionally, to get insight into both the frequency dependence of the modes and the SW profiles in the long-wavelength limit, micromagnetic simulations have been carried out using the MUMAX ${ }^{3}$ code [61]. Here, a magnetic film was built up in the $(64 ; y ; 4096)$ cells with total dimensions of $\left(256 \mathrm{~nm} ; Y_{c}\right.$; $5.3 \mu \mathrm{m})$ and a mesh size of $\left(4.0 \mathrm{~nm} ; Y_{c} ; 3.74 \mathrm{~nm}\right)$. Next, a 140-nm-wide wire with thicknesses of 1-5 nm was centrally put on top of the film, forming the intact film part. The wire was repeated 51 times with a spacing of $a_{z}=300 \mathrm{~nm}$. To consider the reality of an extended surface-modulated film, periodic boundary conditions were chosen along the $x$ and $z$ directions. The number of $y$ cells and the corresponding cell size $Y_{c}$ were chosen according to the ratio of the wire thickness and the film thickness. The external field was applied in the $z$ direction, whereas the excitation field was chosen in the $y$ direction. Two different kinds of simulations were carried out using the system described above. First, the spin-wave dispersion relation was calculated similarly to the approach presented in Ref. [62], but the homogeneous excitation field was replaced by a localized $\mathrm{rf}$ field of approximately $140 \mathrm{~nm}$ width. The spin-wave dispersion was extracted using a spatial fast Fourier transform (FFT) approach along the $z$ direction. Furthermore, FMR simulations based on the approach given in Ref. [63] were carried out. The dynamic magnetization $\mathbf{m}(\mathbf{r}, \omega)$ was extracted by employing a FFT in time for all cells. To obtain the SW profiles for a given frequency $f_{0}$ the transformed amplitude and phase were filtered and then transformed back into real space. Especially in the backward-volume geometry, this approach is very useful in the system presented here due to the more or less flat spin-wave branches. In Fig. 2(f) it is clearly visible that the main intensity is held by the flat branches, making the analysis of higher-order spin waves complicated. In the FMR approach this issue is solved due to the fact that only $k_{z}=0$ modes are excited. For the field-frequency dependence shown in Fig. 4, this approach was repeated for magnetic fields from 0 Oe to $2 \mathrm{kOe}$ in $10-\mathrm{Oe}$ steps. For this purpose the FFT of the average magnetization component was considered. The magnetic parameters are the same as those used in the analytical approach, with a damping constant $\alpha=10^{-2}$.

\section{RESULTS AND DISCUSSION}

The theoretical model will now be applied to thin films with one-dimensional stripelike modulations, as shown in Fig. 1. For such geometry, the Fourier coefficients are given by $C_{G_{n}}=\left(w_{z} / a_{z}\right) \operatorname{sinc}\left[\left(w_{z} / a_{z}\right) \pi n\right]$. Also, at 50 reciprocal lattice vectors, a convergence of the numerical solutions of 

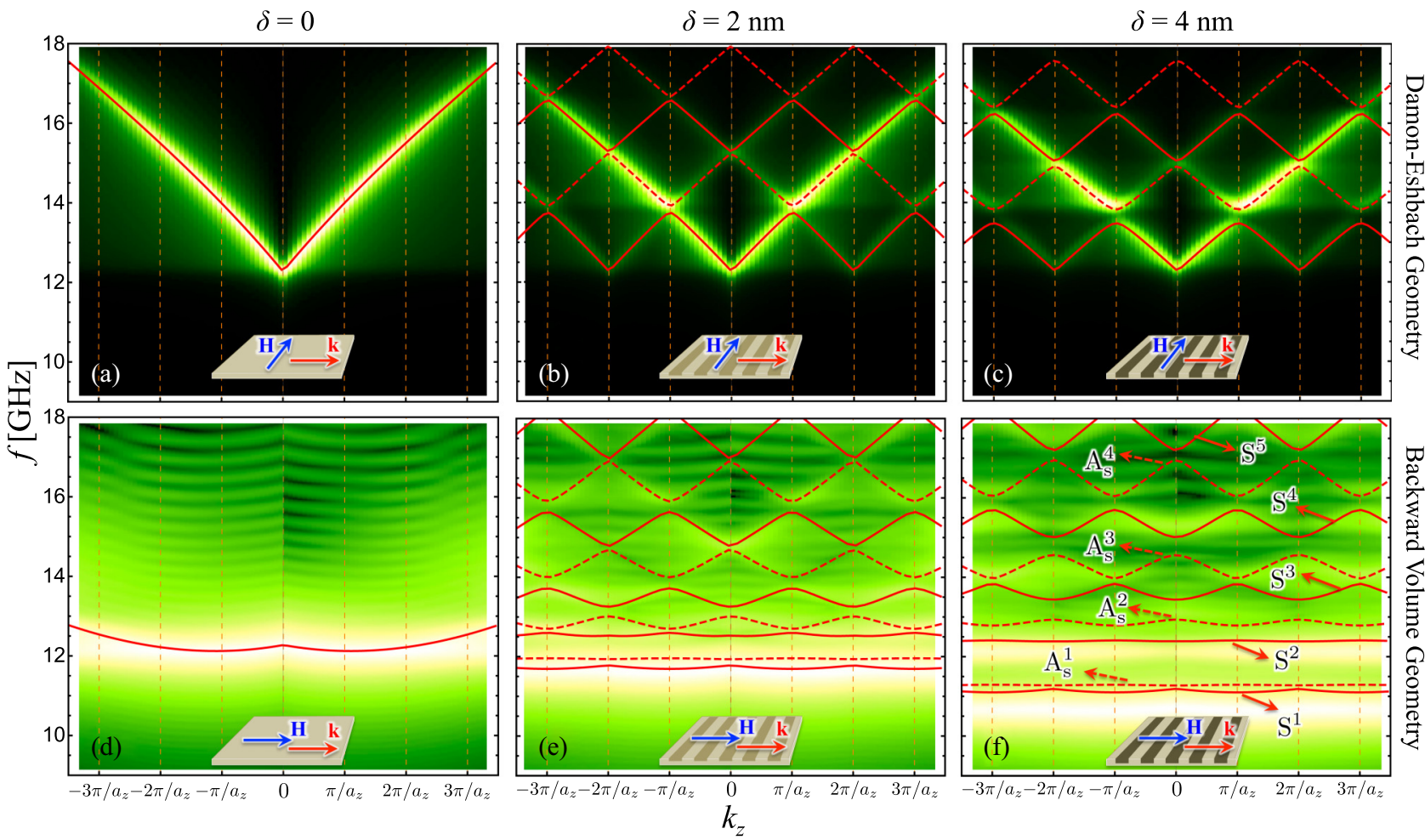

FIG. 2. (a), (b), and (c) show the dispersion relation in the DE geometry for $H=1.5 \mathrm{kOe}$ and $\delta=0,2$, and $4 \mathrm{~nm}$, respectively. (d), (e), and (f) depict the dispersion in the BV configuration. The lines correspond to the theoretical results, while the micromagnetic simulations are represented by the color code, where the lighter (darker) color represents a maximum (minimum) of the response intensity. The modes are labeled $\mathrm{S}^{v}$ and $\mathrm{A}_{\mathrm{s}}^{v}$, with $v=1,2,3, \ldots$ The label $\mathrm{S}^{v}\left(\mathrm{~A}_{\mathrm{s}}^{v}\right)$ is defined according to the symmetric (antisymmetric) character of the spin-wave profiles around $z=0$ at $k_{z}=0$. In the insets, the increasing of the depth $\delta$ is represented as a darker color in the grooves. Due to the finite window size in the FFT additional higher harmonics can be observed in (d)-(f).

Eq. (12) is reached. Typical permalloy (Py) parameters are used, namely, a saturation magnetization $M_{\mathrm{s}}=797 \mathrm{emu} / \mathrm{cm}^{3}$, stiffness constant $\lambda_{\mathrm{ex}}=4.96 \mathrm{~nm}$, and gyromagnetic ratio $\gamma=$ $0.0185556 \mathrm{GHz} / \mathrm{G}$. The thickness of the thicker part $(d+\delta)$ of the SMMC will be assumed to be constant, $d+\delta=10 \mathrm{~nm}$ [see Fig. 1(b)]. So the thinner part of the film is given by $d=10 \mathrm{~nm}-\delta$. Also $a_{z}=300 \mathrm{~nm}$, and $w_{z}=140 \mathrm{~nm}$. Unless otherwise stated, the external field is $H=1.5 \mathrm{kOe}$. Note that this field is strong enough to satisfy the condition $\varphi \approx \varphi_{H}$. These parameters have been chosen in concordance with the micromagnetic simulations.

In Fig. 2, the spin-wave dispersion relations are depicted in both Damon-Eshbach (DE) and backward-volume geometries, in which the depth $\delta$ of the surface modulation is varied. The color code visualizes the micromagnetic simulations, while the lines are obtained from the theoretical model. In Figs. 2(a), 2(b) and 2(c), dispersion relations in the DE geometry $(\varphi=\pi / 2)$ are shown for $\delta=0,2$, and $4 \mathrm{~nm}$, respectively. Here, it is clearly visible that the periodic stray fields created by the dynamic magnetic charges [see Eqs. (10) and (11)] open frequency BGs, whose strength can be controlled through the depth $\delta$. In Figs. 2(d)-2(f) the BV spin waves are shown. Unlike the results of DE geometry, the SWs reveal a slightly dispersive branch at $\delta=0$. Then, by increasing $\delta$ low-frequency flat modes are observed, while the high-frequency ones have a clear periodic dispersion. According to the calculations and micromagnetic simulations, the flat modes should be detectable by experimen- tal techniques such as ferromagnetic resonance (FMR), which should be sufficient to determine the spin-wave modes and their frequency-field dependence, similar to the periodic structures created by ion irradiation $[38,42]$. Note that for $\delta \geqslant 4 \mathrm{~nm}$ in Fig. 2(f) some deviations between simulations and calculations can be noticed. These deviations can be attributed to the nonuniform distribution of the dynamic magnetization along the thickness. While $\delta$ increases, in the BV configuration the creation of magnetostatic charges at the edges of the grooves also increases, so that the magnetization in the corrugated area tends to stay aligned along the stripelike surface modulation. Nevertheless, the magnetic moments in the nominal film of thickness $d$ are more likely to follow the applied field, and hence, they can be easily aligned with the field. Therefore, under a noticeable increase in $\delta$, the magnetization should vary along the normal axis $y$ due to the pinning conditions at the edges of the grooves. Because this modulation is not considered in the theoretical model, some deviations between both methods are expected as $\delta$ increases. On the other hand, due to the finite window size in the FFT, additional higher harmonics can be observed in Figs. 2(d)-2(f). This leakage of the main mode is strongly visible in the BV geometry due to the log-scale plotting.

Figure 3(a) shows the evolution of the first four modes, $S^{1}, S^{2}, S^{3}$, and $S^{4}$, as a function of $\delta$ at $k_{z}=0$. Here, it is observed that the theoretical model (lines) matches very well with the numerical simulations (symbols) in the range 


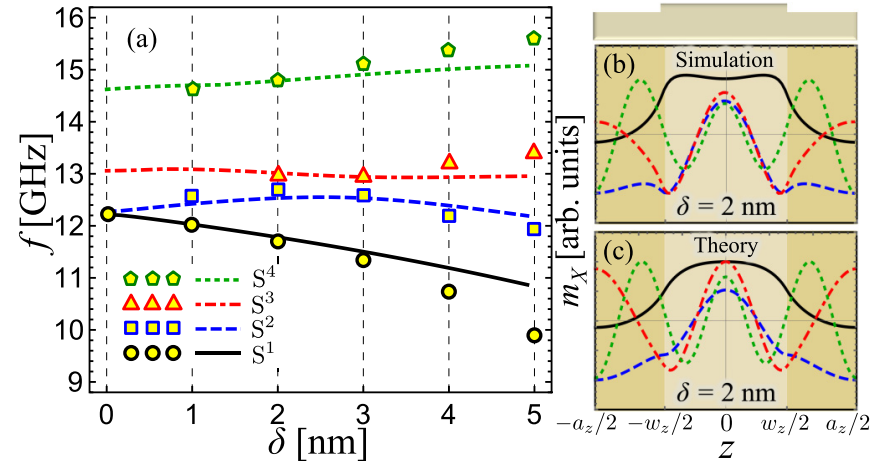

FIG. 3. In (a), the BV modes at $k_{z}=0$ are shown as a function of $\delta$. The lines (symbols) represent the theoretical (simulated) calculations. In (b) and (c) the spin-wave profiles calculated with the simulations and theoretical model are depicted, respectively. In (a)-(c), the symmetric modes $S^{1}, S^{2}, S^{3}$, and $S^{4}$ are represented by solid, dashed, dot-dashed, and dotted lines, respectively.

of $\delta \rightarrow 0-3 \mathrm{~nm}$. For higher depths, small deviations between theory and simulations are observed, as explained above. The simulated and calculated dynamic magnetization profiles of $m_{X}$ at $\delta=2 \mathrm{~nm}$ are illustrated in Figs. 3(b) and 3(c), respectively. Here, there is very good agreement between the two methods, thus corroborating the approximations used in the theoretical model. Overall, it is observed that band gaps are opened by the dynamic dipole fields created by the dynamic magnetizations in the DE configuration, where the BG width can be controlled via variation of $\delta$ since at higher $\delta$ the dipolar field induced by dynamic magnetic charges becomes higher as well. On the other hand, in the backward-volume configuration, the high-frequency BGs and flat modes are mainly induced by static magnetic charges. Because these static magnetic charges are induced by the static magnetization (perpendicular to the stripelike modulations), they have an appreciable effect on the SW dynamics; that is, the SWs are practically confined to some regions of the SMMC, as shown in Figs. 3(b) and 3(c).

Figure 4 shows a comparison between theory and numerical simulations of the FMR response, where the evolution of the modes as a function of the field $H$ is shown. The simulated calculations are given by the color code, whereas dashed lines represent the theoretical results. Here, only the first four modes, $S^{1}, S^{2}, S^{3}$, and $S^{4}$, are depicted since from the point of view of the simulations, the excitation of antisymmetric modes requires an inhomogeneous excitation, which is not included in the micromagnetic code. Similar resonance spectra consisting of the symmetric modes were previously obtained by using linear response theory and two-magnon scattering in the limit of perturbative modulation depths $[36,38]$ but restricted to the $k_{z}=0$ limit.

The flatness of the magnonic modes, the frequency separation between them, as well as their shift to low frequencies represent some of the main findings of this paper. These characteristics can be explained by analyzing the internal field of the periodic structure. For spin waves traveling in the DE geometry, the exchange energy is dominant in the dispersion, and therefore, the frequency rapidly increases with the increasing of the wave vector. Nevertheless, in the BV geometry the spin waves are dominated by the exchange and

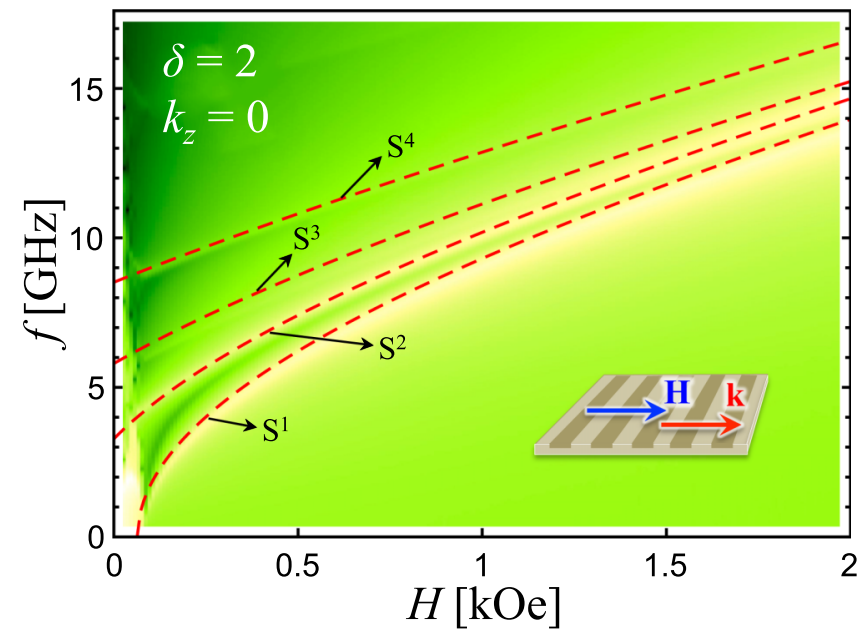

FIG. 4. Frequency as a function of the field for $\delta=2 \mathrm{~nm}$ and $k_{z}=0$. The color code illustrates the simulated data, where the lighter (darker) color represents a maximum (minimum) of the response intensity. The lines correspond to the theoretical results for the first four symmetric modes.

dipolar interaction at small wave vectors, and a nearly flat dispersion is obtained [see Fig. 2(d)]. One can observe that this flatness of the low-frequency modes is enhanced with the patterning. As a consequence of the divergent internal energy landscape generated by the demagnetizing field [see Fig. 5(a)], local maxima and minima lead to a confinement of the spin waves in separated parts of the $\mathrm{MC}$, where the internal energy is minimal. Due to the natural connection of propagation and dispersion, this leads to a spin-wave localization [standing spin waves are depicted in Fig. 5(b)], which is the mechanism causing the flatness of the $f(k)$ dependence of such modes.

Regarding the shift towards low frequencies in the BV geometry, this effect is attributed to the static internal field, which in the case of an SMMC is $H^{\text {int }}=H_{Z}^{\mathrm{I} 0}$. Since the lowfrequency mode $S^{1}$ is located at the center of the thicker part, it experiences a demagnetizing internal field $\left(H^{\text {int }}<0\right)$ that reduces the energy of the mode [see Figs. 5(a) and 5(b)]. Thus, the low-frequency mode in the BV configuration is shifted to low frequencies, as shown in Figs. 5(c) and 5(d). Note that due to this shift towards low frequencies, the flat modes are notably separated in frequency. On the other side, the higher-frequency modes are located partially in the thicker and thinner parts, and hence, they are not shifted to low frequencies. These dynamic properties obtained in an SMMC composed of stripelike grooves can be notoriously different from those of other kinds of MCs. For instance, in bicomponent MCs the static internal field in the BV configuration consists of exchange fields that are induced by the contrast between the exchange constant between the materials and magnetizing/demagnetizing fields that are superimposed on each other because both magnetic materials create magnetic charges at the interfaces. In a bicomponent MC (see Appendix C for details) composed of Py $/$ Co (for cobalt $M_{s}=1150 \mathrm{emu} / \mathrm{cm}^{3}$ and $\lambda_{\mathrm{ex}}=5.89 \mathrm{~nm}$ ) the resulting internal field does not manifest a demagnetizing character in such a way that the low-frequency modes remain in the same frequency position in both DE and BV configurations, as shown in Fig. 5(g) and 5(h). Also, since the modes with 


\section{SURFACE-MODULATED MAGNONIC CRYSTAL}
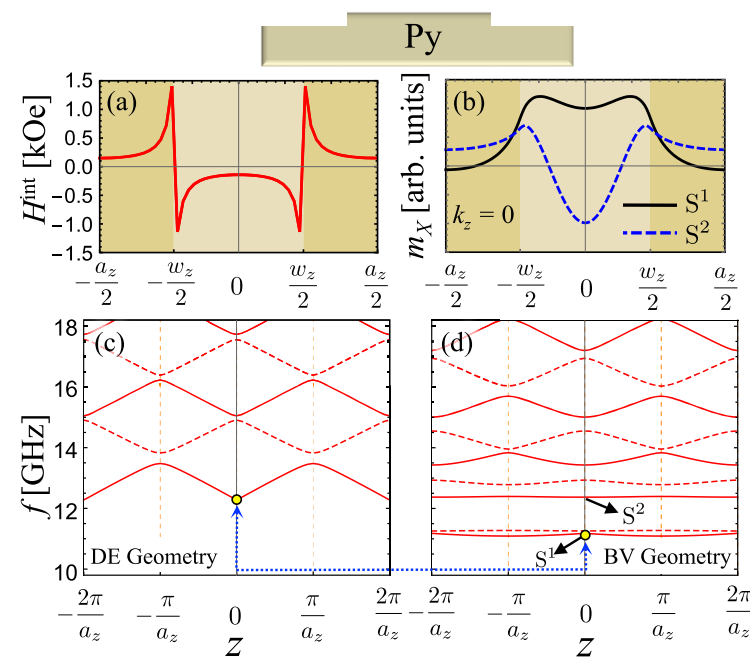

BI-COMPONENT Py/Co MAGNONIC CRYSTAL
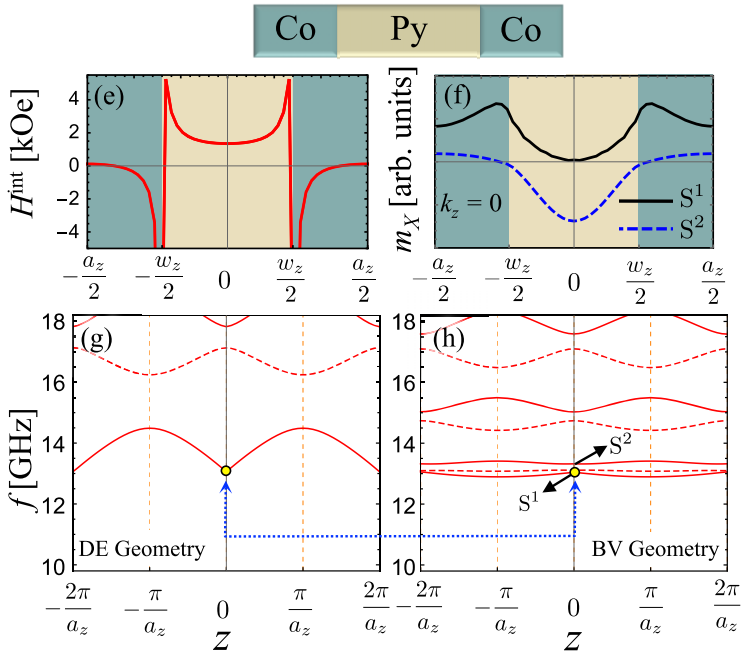

FIG. 5. (a) and (b) show, respectively, the internal field $H^{\text {int }}$ and the SW profile of modes $\mathrm{S}^{1}$ and $\mathrm{S}^{2}$ evaluated at $k_{z}=0$ for an SMMC of $\delta=4 \mathrm{~nm}$. DE and BV spin-wave configurations are depicted in (c) and (d), respectively. (e)-(h) show results for a bicomponent MC composed of alternating Py/Co magnetic materials. The internal field is shown in (e), while the profiles of modes $\mathrm{S}^{1}$ and $\mathrm{S}^{2}$ evaluated at $k_{z}=0$ are illustrated in (f). DE and BV spin-wave configurations are illustrated in (g) and (h), respectively. The dot in the dispersion relations illustrates the low-frequency mode at $k_{z}=0$, which is shifted to low frequencies for an SMMC, while it remains in the same frequency position for a bicomponent MC.

low frequencies are not able to decrease in energy, they are relatively closer to each other [see Fig. 5(h)].

All the calculations presented in Sec. II show a symmetric dispersion under the inversion of the wave vector, despite the fact that the elements $\mathbf{A}_{\mathbf{G}, \mathbf{G}^{\prime}}^{X X}$ and $\mathbf{A}_{\mathbf{G}, \mathbf{G}^{\prime}}^{Y Y}$ [see Eq. (B1a)] are dependent on the sign of the wave vector through function $\xi(\mathbf{G}, \mathbf{k})$, defined in Eq. (5). Therefore, two counterpropagating SWs exhibit a full frequency reciprocity, namely, $f(\mathbf{k})=$ $f(-\mathbf{k})$. This is not surprising since if $\mathbf{A}_{\mathbf{G}, \mathbf{G}^{\prime}}^{X X}=-\mathbf{A}_{\mathbf{G}, \mathbf{G}^{\prime}}^{Y Y}$, the dispersion relation of spin waves depends on the square of $\xi(\mathbf{G}, \mathbf{k})$. Nevertheless, if the dynamic components of the magnetization vary along the thickness, like for the so-called first perpendicular standing SW mode, for instance, the SW frequency becomes dependent on the wave-vector orientation, and nonreciprocal features appear, i.e., $f(\mathbf{k}) \neq f(-\mathbf{k})$, since the condition $\mathbf{A}_{\mathbf{G}, \mathbf{G}^{\prime}}^{X X}=-\mathbf{A}_{\mathbf{G}, \mathbf{G}^{\prime}}^{Y Y}$ is broken. This effect has been observed in Refs. [64,65] for FM films with different magnetic (or geometrical) properties on top and bottom surfaces, where, basically, the symmetry is broken along the thickness by introducing different magnetic anisotropies at the surfaces [64] and by considering antiferromagnetic states between the magnetization in the grooves $(d+\delta<y<d)$ and the bottom FM film of thickness $d$ [65]. Note that in the one-dimensional case $\xi(\mathbf{G}, \mathbf{k})=\left(G_{n}+k_{z}\right) \sin \varphi$, and therefore, the nonreciprocal properties could be enhanced in the Damon-Eshbach geometry $\left(\varphi=90^{\circ}\right)$, such as in the case of spin waves propagating in FM/heavy-metal alloys, where the Dzyaloshinskii-Moriya interaction is important [66-71].

\section{CONCLUSIONS}

Spin waves in surface-modulated magnonic crystals were theoretically modeled using the plane-wave method and micromagnetic simulations. The theory shows that the dipolar interaction produced by surface geometrical modulation is capable of opening magnonic band gaps in either the backward-volume or Damon-Eshbach configuration, where the magnitude of the band gap can be controlled by the geometry of the grooves. An interesting evolution of the modes is found in the backwardvolume configuration, in which flat modes at low frequencies are obtained and whose frequency position can be controlled by the depth of the grooves. Such nearly flat magnonic bands are explained by analyzing the role of the internal field of the SMMC and the spin-wave localization. The theoretical results show that SMMCs can serve as interesting magnonic devices capable of manipulating the spin-wave properties, such as band gaps, spin-wave localization, flat modes, etc., by means of the depth of the groove, in contrast to the typical bicomponent magnonic crystals, where the magnetic properties vary over the entire volume of the magnetic system with a constant film thickness of the film. The approach agrees very well with numerical simulations, which allows us to validate the theoretical findings. Consequently, the model applied to FM films with periodic grooves on top provides further key information about band gap modification, spatial localization of the modes, and the dispersion of the spin waves. Therefore, the results obtained in this work offer a better understanding of such systems, paving the way for further developments in MC-based devices.

\section{ACKNOWLEDGMENTS}

The authors acknowledge financial support from CONICYT PAI/ACADEMIA 79140033, CONICYT/DAAD PCCI140051, FONDECYT 1161403, FONDECYT 3170647, FONDECYT Iniciacion 11170736, and the Centers of Excellence with Basal/CONICYT financing, Grant No. FB0807, CEDENNA. Funding from the Deutsche Forschungsgemeinschaft (Grants No. LE2443/5-1 and No. GE1202/9-2) and DAAD PPP ALECHILE (Grant No. 57136331) are gratefully acknowledged. T.S. acknowledges 
funding from the InProTUC scholarship. M.L. gratefully acknowledges funding from the European Union's Horizon 2020 research and innovation programme under the Marie Skłodowska-Curie Grant Agreement No. 701647.

\section{APPENDIX A: DIPOLAR FIELD INDUCED BY THE GROOVES}

In order to obtain the static periodic field $\mathbf{H}^{\mathrm{I} 0}(\mathbf{r})$, it is noted that the static magnetization components in the range $d<y<$ $d+\delta$ can be written as

$$
M_{z}=M_{\mathrm{s}} \cos \varphi \sum_{\mathbf{G}} C_{\mathbf{G}} \exp [i \mathbf{G} \cdot \mathbf{r}]
$$

and

$$
M_{x}=M_{\mathrm{s}} \sin \varphi \sum_{\mathbf{G}} C_{\mathbf{G}} \exp [i \mathbf{G} \cdot \mathbf{r}] .
$$

Note that these magnetization components are null for $y^{\prime}<d$ and $y>d+\delta$, which indicates that the magnetic charges will be present only in the zone of the grooves. Then, following Ref. [38], the magnetostatic potential is given by

$$
\phi(\mathbf{r})=-i M_{\mathrm{s}} \sum_{\mathbf{G}} \chi(\mathbf{G}) \int C_{\mathbf{G}} \frac{e^{i \mathbf{G} \cdot \mathbf{r}^{\prime}}}{\left|\mathbf{r}-\mathbf{r}^{\prime}\right|} \mathrm{d}^{3} \mathbf{r}^{\prime},
$$

where $\chi(\mathbf{G})=G_{n} \cos \varphi+G_{q} \sin \varphi$. Therefore, an analytical expression can be derived for the magnetostatic potential, which is

$$
\phi_{1}(\mathbf{r})=i 2 \pi M_{\mathrm{s}} \sum_{\mathbf{G}} C_{\mathbf{G}} \chi(\mathbf{G}) \frac{e^{|\mathbf{G}|(y-d-\delta)}\left(1-e^{|\mathbf{G}| \delta}\right)}{|\mathbf{G}|^{2}} e^{i \mathbf{G} \cdot \mathbf{r}}
$$

for $0<y<d$ and

$$
\begin{aligned}
\phi_{2}(\mathbf{r})= & i 4 \pi M_{\mathrm{S}} \sum_{\mathbf{G}} C_{\mathbf{G} \chi}(\mathbf{G}) \\
& \times \frac{1-\cosh [|\mathbf{G}|(d+\delta / 2-y)] e^{|\mathbf{G}| \delta / 2}}{|\mathbf{G}|^{2}} e^{i \mathbf{G} \cdot \mathbf{r}}
\end{aligned}
$$

for the potential in the zone $d<y<d+\delta$. Thus, the $Z$ component of the static magnetic field is $H_{Z}^{\mathrm{I} 0}(\mathbf{r})=H_{1 Z}^{\mathrm{I} 0}(\mathbf{r})+$ $H_{2 Z}^{\mathrm{I0}}(\mathbf{r})$, with $H_{1 Z}^{\mathrm{I0}}(\mathbf{r})\left[H_{2 Z}^{\mathrm{I0}}(\mathbf{r})\right]$ being the static field inside the zone $d>y>0[d+\delta>y>d]$. Then, the field can be obtained from $\mathbf{H}_{1}^{\mathrm{I} 0}(\mathbf{r})=-\nabla_{\mathbf{r}} \phi_{1}$ and $\mathbf{H}_{2}^{\mathrm{I} 0}(\mathbf{r})=-\nabla_{\mathbf{r}} \phi_{2}$, whose $Z$ components are given in Eqs. (7) and (8).

In expressions (7) and (8), an average over the thickness $d+\delta$ has been performed. Note that the magnetic field $H_{1 Z}^{\mathrm{I} 0}(\mathbf{r})$ dominates when the thickness $\delta$ is small; nevertheless, when $\delta$ increases, the field $H_{2 Z}^{\mathrm{I} 0}(\mathbf{r})$ becomes important. Thus, it is expected to represent surface-modulated magnonic crystals with significant depths. On the other hand, the dynamic magnetization components in the zone $d+\delta>y>d$ can be written as

$$
m_{X, Y}(\mathbf{r})=\sum_{\mathbf{G}, \mathbf{G}^{\prime}} m_{X, Y}(\mathbf{G}) C_{\mathbf{G}^{\prime}} e^{i\left(\mathbf{G}+\mathbf{G}^{\prime}+\mathbf{k}\right) \cdot \mathbf{r}},
$$

where it is assumed that this dynamic magnetization is uniform along the thickness. This approximation is valid for small values of depth $\delta$; nevertheless, when $\delta$ increases, the boundary conditions may produce a modulation of spin waves along the thickness, and therefore, Eq. (A4) is not valid anymore. By using the same procedure to derive Eqs. (7) and (8), the dynamic components shown in Eqs. (10) and (11) are obtained.

\section{APPENDIX B: MATRIX ELEMENTS FOR A SMMC}

The matrix elements in Eq. (13) are given by

$$
\begin{aligned}
& \mathbf{A}_{\mathbf{G}, \mathbf{G}^{\prime}}^{X X}=-\mathbf{A}_{\mathbf{G}, \mathbf{G}^{\prime}}^{Y Y}=-i 2 \pi M_{\mathrm{s}} C_{\mathbf{G}-\mathbf{G}^{\prime}} \xi(\mathbf{G}, \mathbf{k}) \frac{\eta(\mathbf{G}+\mathbf{k})}{|\mathbf{G}+\mathbf{k}|} \\
& \mathbf{A}_{\mathbf{G}, \mathbf{G}^{\prime}}^{X Y}=-\left[4 \pi M_{\mathrm{s}} \lambda_{\mathrm{ex}}^{2}(\mathbf{G}+\mathbf{k})^{2}+4 \pi M_{\mathrm{s}} \zeta(\mathbf{G}+\mathbf{k}, d)+H\right] \delta_{\mathbf{G}, \mathbf{G}^{\prime}}+2 \pi M_{\mathrm{s}} C_{\mathbf{G}-\mathbf{G}^{\prime}} F_{X Y}^{\mathrm{I}}+\Psi\left(\mathbf{G}^{\prime}, \mathbf{G}\right) \\
& \mathbf{A}_{\mathbf{G}, \mathbf{G}^{\prime}}^{Y X}=\left[4 \pi M_{\mathrm{s}} \lambda_{\mathrm{ex}}^{2}(\mathbf{G}+\mathbf{k})^{2}+4 \pi M_{\mathrm{s}} \xi(\mathbf{G}, \mathbf{k})^{2} \frac{1-\zeta(\mathbf{G}+\mathbf{k}, d)}{|\mathbf{G}+\mathbf{k}|^{2}}+H\right] \delta_{\mathbf{G}, \mathbf{G}^{\prime}}+2 \pi M_{\mathrm{s}} C_{\mathbf{G}-\mathbf{G}^{\prime}} F_{Y X}^{\mathrm{I}}-\Psi\left(\mathbf{G}^{\prime}, \mathbf{G}\right) .
\end{aligned}
$$

Here, it has been assumed that the external field is strong enough to orient the magnetization parallel to it. Also, functions $F_{X Y}^{\mathrm{I}}$ and $F_{Y X}^{\mathrm{I}}$ come from the dipolar interaction induced by the grooves and are given by

$$
F_{X Y}^{\mathrm{I}}=\eta(\mathbf{G}+\mathbf{k})+\frac{\chi\left(\mathbf{G}-\mathbf{G}^{\prime}\right)^{2}}{\left|\mathbf{G}-\mathbf{G}^{\prime}\right|^{2}} \frac{\eta\left(\mathbf{G}-\mathbf{G}^{\prime}\right) d}{d+\delta}
$$

and

$$
F_{Y X}^{\mathrm{I}}=\xi(\mathbf{G}, \mathbf{k})^{2} \frac{\eta(\mathbf{G}+\mathbf{k})}{|\mathbf{G}+\mathbf{k}|^{2}}-\frac{\chi\left(\mathbf{G}-\mathbf{G}^{\prime}\right)^{2}}{\left|\mathbf{G}-\mathbf{G}^{\prime}\right|^{2}} \frac{\eta\left(\mathbf{G}-\mathbf{G}^{\prime}\right) d}{d+\delta},
$$

while function $\Psi_{\mathbf{G}^{\prime}, \mathbf{G}}$ comes from the static stray field $H_{2 Z}^{\mathrm{I} 0}(\mathbf{r})$ that interacts with the magnetization of the grooves. This function is

$$
\Psi\left(\mathbf{G}^{\prime}, \mathbf{G}\right)=\frac{4 \pi M_{\mathrm{s}} \delta}{d+\delta} \sum_{\mathbf{G}^{\prime \prime}} C_{\mathbf{G}^{\prime \prime}-\mathbf{G}^{\prime}} C_{\mathbf{G}-\mathbf{G}^{\prime \prime}} \frac{\chi\left(\mathbf{G}^{\prime \prime}-\mathbf{G}^{\prime}\right)^{2}}{\left|\mathbf{G}^{\prime \prime}-\mathbf{G}^{\prime}\right|^{2}}\left[1-\zeta\left(\mathbf{G}^{\prime \prime}-\mathbf{G}^{\prime}, \delta\right)\right] .
$$




\section{APPENDIX C: MATRIX ELEMENTS FOR A BICOMPONENT MC}

In the case of the bicomponent magnonic crystal depicted in Fig. 5, both the saturation magnetization and the exchange length are periodic functions. If the saturation magnetization changes from $M_{\mathrm{s}}$ to $M_{\mathrm{s}}^{\prime}$, then it can be written as

$$
M_{\mathrm{s}}(\mathbf{r})=\sum_{\mathbf{G}} M_{\mathrm{s}}(\mathbf{G}) e^{i \mathbf{G} \cdot \mathbf{r}}
$$

In the same way, the exchange length is

$$
\lambda_{\mathrm{ex}}(\mathbf{r})=\sum_{\mathbf{G}} \lambda_{\mathrm{ex}}(\mathbf{G}) e^{i \mathbf{G} \cdot \mathbf{r}}
$$

since $\lambda_{\text {ex }}=\sqrt{2 A / 4 \pi M_{\mathrm{s}}^{2}}$. Then, the matrix elements in Eq. (13) are given by $\mathbf{A}_{\mathbf{G}, \mathbf{G}^{\prime}}^{X X}=\mathbf{A}_{\mathbf{G}, \mathbf{G}^{\prime}}^{Y Y}=0$,

$$
\begin{aligned}
\mathbf{A}_{\mathbf{G}, \mathbf{G}^{\prime}}^{X Y}= & -H \delta_{\mathbf{G}, \mathbf{G}^{\prime}}+4 \pi M_{\mathrm{s}}\left(\mathbf{G}-\mathbf{G}^{\prime}\right)\left[\chi\left(\mathbf{G}-\mathbf{G}^{\prime}\right)^{2} \frac{1-\zeta\left(\mathbf{G}-\mathbf{G}^{\prime}, d\right)}{\left|\mathbf{G}-\mathbf{G}^{\prime}\right|^{2}}-\zeta\left(\mathbf{G}^{\prime}+\mathbf{k}, d\right)\right] \\
& -4 \pi \sum_{\mathbf{G}^{\prime \prime}} M_{\mathrm{s}}\left(\mathbf{G}-\mathbf{G}^{\prime \prime}\right)\left[\left(\mathbf{G}^{\prime}+\mathbf{k}\right) \cdot\left(\mathbf{G}^{\prime \prime}+\mathbf{k}\right)-\left(\mathbf{G}-\mathbf{G}^{\prime \prime}\right) \cdot\left(\mathbf{G}-\mathbf{G}^{\prime}\right)\right]\left[\lambda_{\mathrm{ex}}\left(\mathbf{G}^{\prime \prime}-\mathbf{G}^{\prime}\right)\right]^{2}, \\
\mathbf{A}_{\mathbf{G}, \mathbf{G}^{\prime}}^{Y X}= & H \delta_{\mathbf{G}, \mathbf{G}^{\prime}}-4 \pi M_{\mathrm{s}}\left(\mathbf{G}-\mathbf{G}^{\prime}\right)\left\{\chi\left(\mathbf{G}-\mathbf{G}^{\prime}\right)^{2} \frac{1-\zeta\left(\mathbf{G}-\mathbf{G}^{\prime}, d\right)}{\left|\mathbf{G}-\mathbf{G}^{\prime}\right|^{2}}+\xi\left(\mathbf{G}^{\prime}, \mathbf{k}\right)^{2}\left[\frac{\zeta\left(\mathbf{G}^{\prime}+\mathbf{k}, d\right)-1}{\left|\mathbf{G}^{\prime}+\mathbf{k}\right|^{2}}\right]\right\} \\
& +4 \pi \sum_{\mathbf{G}^{\prime \prime}} M_{\mathrm{s}}\left(\mathbf{G}-\mathbf{G}^{\prime \prime}\right)\left[\left(\mathbf{G}^{\prime}+\mathbf{k}\right) \cdot\left(\mathbf{G}^{\prime \prime}+\mathbf{k}\right)-\left(\mathbf{G}-\mathbf{G}^{\prime \prime}\right) \cdot\left(\mathbf{G}-\mathbf{G}^{\prime}\right)\right]\left[\lambda_{\mathrm{ex}}\left(\mathbf{G}^{\prime \prime}-\mathbf{G}^{\prime}\right)\right]^{2} .
\end{aligned}
$$

Note that for the one-dimensional case depicted in this paper, the Fourier coefficient of the saturation magnetization is

$$
M_{\mathrm{s}}\left(G_{n}\right)=\left(M_{\mathrm{s}}-M_{\mathrm{s}}^{\prime}\right) \frac{w_{z}}{a_{z}} \operatorname{sinc}\left(G_{n} \frac{w_{z}}{2}\right)+M_{\mathrm{s}}^{\prime} \delta_{n, 0}
$$

The same structure was used for the exchange length coefficient, i.e.,

$$
\lambda_{\mathrm{ex}}\left(G_{n}\right)=\left(\lambda_{\mathrm{ex}}-\lambda_{\mathrm{ex}}^{\prime}\right) \frac{w_{z}}{a_{z}} \operatorname{sinc}\left(G_{n} \frac{w_{z}}{2}\right)+\lambda_{\mathrm{ex}}^{\prime} \delta_{n, 0},
$$

where $\lambda_{\mathrm{ex}}^{\prime}=\sqrt{2 A / 4 \pi\left(M_{\mathrm{s}}^{\prime}\right)^{2}}$.

[1] S. Demokritov and A. Slavin, Magnonics: From Fundamentals to Applications, Topics in Applied Physics, Vol. 125 (Springer, Berlin, 2012).

[2] A. V. Chumak, V. I. Vasyuchka, A. A. Serga, and B. Hillebrands, Nat. Phys. 11, 453 (2015).

[3] M. P. Kostylev, A. A. Serga, T. Schneider, B. Leven, and B. Hillebrands, Appl. Phys. Lett. 87, 153501 (2005).

[4] S. Tacchi, P. Gruszecki, M. Madami, G. Carlotti, J. W. Kłos, M. Krawczyk, A. Adeyeye, and G. Gubbiotti, Sci. Rep. 5, 10367 (2015).

[5] A. Khitun and K. L. Wang, Superlattices Microstruct. 38, 184 (2005).

[6] A. Khitun, M. Bao, and K. L. Wang, J. Phys. D 43, 264005 (2010).

[7] M. Jamali, J. H. Kwon, S.-M. Seo, K.-J. Lee, and H. Yang, Sci. Rep. 3, 3160 (2013).

[8] A. V. Chumak, A. A. Serga, and B. Hillebrands, Nat. Commun. 5, 4700 (2014).

[9] J. O. Vasseur, L. Dobrzynski, B. Djafari-Rouhani, and H. Puszkarski, Phys. Rev. B 54, 1043 (1996).

[10] S. Neusser, B. Botters, and D. Grundler, Phys. Rev. B 78, 054406 (2008).
[11] S. Tacchi, F. Montoncello, M. Madami, G. Gubbiotti, G. Carlotti, L. Giovannini, R. Zivieri, F. Nizzoli, S. Jain, A. O. Adeyeye, and N. Singh, Phys. Rev. Lett. 107, 127204 (2011).

[12] S. Tacchi, G. Duerr, J. W. Klos, M. Madami, S. Neusser, G. Gubbiotti, G. Carlotti, M. Krawczyk, and D. Grundler, Phys. Rev. Lett. 109, 137202 (2012).

[13] Z. K. Wang, V. L. Zhang, H. S. Lim, S. C. Ng, M. H. Kuok, S. Jain, and A. O. Adeyeye, Appl. Phys. Lett. 94, 083112 (2009).

[14] Z. K. Wang, V. L. Zhang, H. S. Lim, S. C. Ng, M. H. Kuok, S. Jain, and A. O. Adeyeye, ACS Nano 4, 643 (2010).

[15] A. V. Chumak, V. I. Vasyuchka, A. A. Serga, M. P. Kostylev, V. S. Tiberkevich, and B. Hillebrands, Phys. Rev. Lett. 108, 257207 (2012).

[16] H. Yu, G. Duerr, R. Huber, M. Bahr, T. Schwarze, F. Brandl, and D. Grundler, Nat. Commun. 4, 2702 (2013).

[17] M. Krawczyk and D. Grundler, J. Phys.: Condens. Matter 26 , 123202 (2014).

[18] S. Nikitov, P. Tailhades, and C. Tsai, J. Magn. Magn. Mater. 236, 320 (2001).

[19] H. Puszkarski and M. Krawczyk, Solid State Phenom. 94, 125 (2003).

[20] V. Kruglyak and R. Hicken, J. Magn. Magn. Mater. 306, 191 (2006). 
[21] G. Gubbiotti, S. Tacchi, G. Carlotti, N. Singh, S. Goolaup, A. O. Adeyeye, and M. Kostylev, Appl. Phys. Lett. 90, 092503 (2007).

[22] M. Krawczyk and H. Puszkarski, Phys. Rev. B 77, 054437 (2008).

[23] A. V. Chumak, P. Pirro, A. A. Serga, M. P. Kostylev, R. L. Stamps, H. Schultheiss, K. Vogt, S. J. Hermsdoerfer, B. Laegel, P. A. Beck, and B. Hillebrands, Appl. Phys. Lett. 95, 262508 (2009).

[24] S. Neusser and D. Grundler, Adv. Mater. 21, 2927 (2009).

[25] A. A. Serga, A. V. Chumak, and B. Hillebrands, J. Phys. D 43, 264002 (2010).

[26] V. V. Kruglyak, S. O. Demokritov, and D. Grundler, J. Phys. D 43, 264001 (2010).

[27] G. Gubbiotti, S. Tacchi, M. Madami, G. Carlotti, A. O. Adeyeye, and M. Kostylev, J. Phys. D 43, 264003 (2010).

[28] Y. Cao, G. Yun, X. Liang, and N. Bai, J. Phys. D 43, 305005 (2010).

[29] B. Lenk, H. Ulrichs, F. Garbs, and M. Münzenberg, Phys. Rep. 507, 107 (2011).

[30] K.-S. Lee, D.-S. Han, and S.-K. Kim, Phys. Rev. Lett. 102, 127202 (2009).

[31] S.-K. Kim, K.-S. Lee, and D.-S. Han, Appl. Phys. Lett. 95, 082507 (2009).

[32] J. Ding, M. Kostylev, and A. O. Adeyeye, Phys. Rev. B 84, 054425 (2011).

[33] T. Sebastian, K. Schultheiss, B. Obry, B. Hillebrands, and H. Schultheiss, Front. Phys. 3, 35 (2015).

[34] J. W. Kłos, D. Kumar, J. Romero-Vivas, H. Fangohr, M. Franchin, M. Krawczyk, and A. Barman, Phys. Rev. B 86, 184433 (2012).

[35] M. Krawczyk, S. Mamica, M. Mruczkiewicz, J. W. Klos, S. Tacchi, M. Madami, G. Gubbiotti, G. Duerr, and D. Grundler, J. Phys. D 46, 495003 (2013).

[36] P. Landeros and D. L. Mills, Phys. Rev. B 85, 054424 (2012).

[37] M. Körner, K. Lenz, R. A. Gallardo, M. Fritzsche, A. Mücklich, S. Facsko, J. Lindner, P. Landeros, and J. Fassbender, Phys. Rev. B 88, 054405 (2013).

[38] R. A. Gallardo, A. Banholzer, K. Wagner, M. Körner, K. Lenz, M. Farle, J. Lindner, J. Fassbender, and P. Landeros, New J. Phys. 16, 023015 (2014).

[39] S. A. Odintsov, A. V. Sadovnikov, A. A. Grachev, E. N. Beginin, Y. P. Sharaevskii, and S. A. Nikitov, JETP Lett. 104, 563 (2016).

[40] M. Langer, F. Röder, R. A. Gallardo, T. Schneider, S. Stienen, C. Gatel, R. Hübner, L. Bischoff, K. Lenz, J. Lindner, P. Landeros, and J. Fassbender, Phys. Rev. B 95, 184405 (2017).

[41] S. Khanal, P. Sherpa, and L. Spinu, IEEE Trans. Magn. 53, 1 (2017).

[42] I. Barsukov, F. M. Römer, R. Meckenstock, K. Lenz, J. Lindner, S. Hemken to Krax, A. Banholzer, M. Körner, J. Grebing, J. Fassbender, and M. Farle, Phys. Rev. B 84, 140410(R) (2011).

[43] B. Obry, P. Pirro, T. Brächer, A. V. Chumak, J. Osten, F. Ciubotaru, A. A. Serga, J. Fassbender, and B. Hillebrands, Appl. Phys. Lett. 102, 202403 (2013).

[44] Q. Wang, A. V. Chumak, L. Jin, H. Zhang, B. Hillebrands, and Z. Zhong, Phys. Rev. B 95, 134433 (2017).

[45] A. V. Chumak, A. A. Serga, S. Wolff, B. Hillebrands, and M. P. Kostylev, J. Appl. Phys. 105, 083906 (2009).
[46] M. Mruczkiewicz, E. S. Pavlov, S. L. Vysotsky, M. Krawczyk, Y. A. Filimonov, and S. A. Nikitov, Phys. Rev. B 90, 174416 (2014).

[47] C. S. Lin, H. S. Lim, Z. K. Wang, S. C. Ng, and M. H. Kuok, Appl. Phys. Lett. 98, 022504 (2011).

[48] M. Sokolovskyy and M. Krawczyk, J. Nanopart. Res. 13, 6085 (2011).

[49] M. Mruczkiewicz, M. Krawczyk, V. K. Sakharov, Y. V. Khivintsev, Y. A. Filimonov, and S. A. Nikitov, J. Appl. Phys. 113, 093908 (2013).

[50] C. G. Sykes, J. D. Adam, and J. H. Collins, Appl. Phys. Lett. 29 , 388 (1976).

[51] A. V. Chumak, A. A. Serga, B. Hillebrands, and M. P. Kostylev, Appl. Phys. Lett. 93, 022508 (2008).

[52] G. R. Aranda, G. N. Kakazei, J. González, and K. Y. Guslienko, J. Appl. Phys. 116, 093908 (2014).

[53] V. D. Bessonov, M. Mruczkiewicz, R. Gieniusz, U. Guzowska, A. Maziewski, A. I. Stognij, and M. Krawczyk, Phys. Rev. B 91 , 104421 (2015).

[54] I. Lisenkov, D. Kalyabin, S. Osokin, J. Klos, M. Krawczyk, and S. Nikitov, J. Magn. Magn. Mater. 378, 313 (2015).

[55] S. L. Vysotskii, Y. V. Khivintsev, V. K. Sakharov, G. M. Dudko, A. V. Kozhevnikov, S. A. Nikitov, N. N. Novitskii, A. I. Stognij, and Y. A. Filimonov, IEEE Magn. Lett. 8, 1 (2017).

[56] M. P. Kostylev, A. A. Stashkevich, and N. A. Sergeeva, Phys. Rev. B 69, 064408 (2004).

[57] I. Lisenkov, D. Kalyabin, and S. Nikitov, Appl. Phys. Lett. 103, $202402(2013)$

[58] J. W. Kłos, M. Krawczyk, and M. Sokolovskyy, J. Appl. Phys. 109, 07D311 (2011).

[59] R. Shindou, R. Matsumoto, S. Murakami, and J.-i. Ohe, Phys. Rev. B 87, 174427 (2013).

[60] I. Lisenkov, V. Tyberkevych, S. Nikitov, and A. Slavin, Phys. Rev. B 93, 214441 (2016).

[61] A. Vansteenkiste, J. Leliaert, M. Dvornik, M. Helsen, F. GarciaSanchez, and B. Van Waeyenberge, AIP Adv. 4, 107133 (2014).

[62] K. Wagner, S. Stienen, and M. Farle, arXiv:1506.05292.

[63] R. D. McMichael and M. D. Stiles, J. Appl. Phys. 97, $10 J 901$ (2005).

[64] O. Gladii, M. Haidar, Y. Henry, M. Kostylev, and M. Bailleul, Phys. Rev. B 93, 054430 (2016).

[65] K. Di, S. X. Feng, S. N. Piramanayagam, V. L. Zhang, H. S. Lim, S. C. Ng, and M. H. Kuok, Sci. Rep. 5, 10153 (2015).

[66] K. Zakeri, Y. Zhang, J. Prokop, T.-H. Chuang, N. Sakr, W. X. Tang, and J. Kirschner, Phys. Rev. Lett. 104, 137203 (2010).

[67] D. Cortés-Ortuño and P. Landeros, J. Phys.: Condens. Matter 25, 156001 (2013).

[68] K. Di, V. L. Zhang, H. S. Lim, S. C. Ng, M. H. Kuok, J. Yu, J. Yoon, X. Qiu, and H. Yang, Phys. Rev. Lett. 114, 047201 (2015).

[69] J. Cho, N.-H. Kim, S. Lee, J.-S. Kim, R. Lavrijsen, A. Solignac, Y. Yin, D.-S. Han, N. J. J. van Hoof, H. J. M. Swagten, B. Koopmans, and C.-Y. You, Nat. Commun. 6, 7635 (2015).

[70] H. T. Nembach, J. M. Shaw, M. Weiler, E. Jue, and T. J. Silva, Nat. Phys. 11, 825 (2015).

[71] S. Tacchi, R. E. Troncoso, M. Ahlberg, G. Gubbiotti, M. Madami, J. Åkerman, and P. Landeros, Phys. Rev. Lett. 118, 147201 (2017). 